\title{
Prognostic and predictive role of hyponatremia in cancer patients
}

\author{
Alessandro Peri \\ Sodium Unit, Endocrinology, Department of Experimental and Clinical Biomedical Sciences "Mario Serio", University of Florence, \\ Careggi Hospital, Florence 50139, Italy. \\ Correspondence to: Prof. Alessandro Peri, Sodium Unit, Endocrinology, Department of Experimental and Clinical Biomedical \\ Sciences "Mario Serio", University of Florence, Careggi Hospital, AOU Careggi, Viale Pieraccini 6, Florence 50139, Italy. \\ E-mail: alessandro.peri@unifi.it
}

How to cite this article: Peri A. Prognostic and predictive role of hyponatremia in cancer patients. $J$ Cancer Metastasis Treat 2019;5:40. http://dx.doi.org/10.20517/2394-4722.2019.14

Received: 5 Feb 2019 First Decision: 25 Feb 2019 Revised: 4 Mar 2019 Accepted: 11 Mar 2019 Published: 6 May 2019

Science Editor: Berardi Rossana Copy Editor: Cai-Hong Wang Production Editor: Huan-Liang Wu

\begin{abstract}
Hyponatremia is the most frequent electrolyte disorder encountered in hospitalized patients. Several studies have demonstrated that hyponatremia is a negative prognostic factor in different clinical scenarios. Noteworthy, not only severe and acute hyponatremia has been associated with an increased risk of mortality, but also moderate of even mild chronic hyponatremia may increase the risk of death. This has been demonstrated in different categories of patients, including cancer patients. There is growing evidence that both progression free survival and overall survival are significantly reduced in cancer patients with hyponatremia compared to patients with normonatremia. One important, and still open, question is whether the worse outcome associated with hyponatremia in cancer patients is directly attributable to the electrolyte disorder itself or might be a sign of the severity of the underlying disease. With regard to this point, some basic research studies suggested that low sodium concentration stimulates per se cancer cells proliferation and invasiveness. Recent clinical evidence appears to indicate that the correction of hyponatremia is an independent and favourable prognostic factor in cancer patients. Admittedly, robust confirmatory data from clinical practice are needed, in order to validate the hypothesis that cancer patients may die for hyponatremia and not just with hyponatremia.
\end{abstract}

Keywords: Hyponatremia, cancer, mortality, prognosis, vasopressin

\section{INTRODUCTION}

Hyponatremia, which is the most frequent electrolyte disorder in hospitalized patients ${ }^{[1]}$, frequently occurs also in cancer patients at different stages of disease. For instance, a recent retrospective study, performed

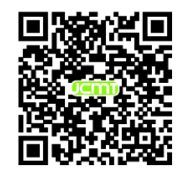


at a large University Hospital in Germany in inpatients receiving specialist palliative care, showed that the prevalence of hyponatremia was $38.7 \%$ (e.g., 275 out of 710 patients) and that low serum sodium concentration $\left[\mathrm{Na}^{+}\right]$was associated with symptom severity ${ }^{[2]}$. Several studies have demonstrated that hyponatremia is a negative prognostic factor in different clinical scenarios. Noteworthy, not only severe and acute hyponatremia has been associated with an increased risk of mortality, but there is evidence that also moderate of even mild chronic hyponatremia may increase the risk of death. This has been shown for instance in a large cohort study, which included more than 50,000 inpatients at a teaching academic medical center. This study showed that mildly reduced serum $\left[\mathrm{Na}^{+}\right]$was associated with an increased in-hospital mortality. In addition, each mmol/L reduction of serum $\left[\mathrm{Na}^{+}\right]$increased the risk of death by $2.3 \%{ }^{[3]}$. Among different clinical settings, hyponatremia has been associated with an increased risk of death in patients with heart failure ${ }^{[4-6]}$, acute myocardial infarction ${ }^{[7,8]}$, pneumonia ${ }^{[9]}$, cirrhosis $^{[10,11]}$, renal failure ${ }^{[12]}$, pulmonary embolism $^{[13]}$, intracerebral hemorrhage ${ }^{[14]}$, in the elderly ${ }^{[15]}$, in intensive care patients ${ }^{[16]}$, in patients undergoing surgery ${ }^{[17]}$ or cardiovascular procedures ${ }^{[18]}$. An extensive meta-analysis, including more than 850,000 patients, of whom $17.4 \%$ with hyponatremia, confirmed an increased mortality rate associated with reduced serum $\left[\mathrm{Na}^{+}\right]^{[19]}$.

The literature addressing cancer patients indicated that hyponatremia is a negative prognostic factor also in this subset of patients. This chapter will review the published data, so far, with the exception of patients with lung cancer, which is the specific topic of another chapter.

\section{HYPONATREMIA AS A PROGNOSTIC AND PREDICTIVE FACTOR IN CANCER PATIENTS}

Ten years ago Waikar et al. ${ }^{[7]}$ published the results of a prospective cohort study, conducted in two teaching hospitals in Boston, MS, in which the association between serum $\left[\mathrm{Na}^{+}\right]$and mortality was investigated. The main outcome measures were in-hospital 1-year and 5-year mortality of patients with hyponatremia $v s$ those with normal serum $\left[\mathrm{Na}^{+}\right]$. Patients with hyponatremia had higher in-hospital mortality rates either at 1 year and at 5 years, even when serum $\left[\mathrm{Na}^{+}\right]$was only slightly reduced $(130-134 \mathrm{mmol} / \mathrm{L})$. Among the different clinical subgroups that were analyzed, the risk of in-hospital mortality was significantly higher in hyponatremic patients with metastatic cancer [hazard ratio (HR) 2.05, 95\% confidence interval (CI): 1.672.53] compared to normonatremic patients.

Shortly after, another study addressed predictors of inpatients mortality in an acute palliative care unit at the M. D. Anderson Cancer Center of the University of Texas. Of the 500 cancer patients admitted, 124 (25\%) $\operatorname{died}^{[20]}$. Hyponatremia was one of the multiple predictors of a negative outcome (HR 3.02, 95\%CI: 1.76-5.17, $P<$ 0.001), together with younger age, hypernatremia, high blood urea nitrogen, high heart rate, high respiration rate, and supplemental oxygen use.

A retrospective analysis of patients admitted at the same Cancer Center during a three months period in 2006, aiming to correlate hyponatremia with 90 -day mortality, was performed ${ }^{[21]}$. Serum $\left[\mathrm{Na}^{+}\right]$was categorized into four groups, e.g., normonatremia (serum $\left[\mathrm{Na}^{+}\right]$135-147 mmol/L), mild (130-134 mmol/L), moderate (120-129 mmol/L), and severe $(<120 \mathrm{mmol} / \mathrm{L})$ hyponatremia. In all hyponatremic groups the risk of mortality at 90 days was higher than in normonatremic patients (HR for mild, moderate, and severe hyponatremia: 2.04 (95\%CI: 1.42-2.91; $P<0.01$ ); 4.74 (95\%CI: 3.21-7.01; $P<0.01$ ), and 3.46 (95\%CI: 1.05-11.44; $P=0.04$ ), respectively. Although the authors recognized limitations to the study (observational, retrospective study, inability to adjust for all comorbid conditions), and the fact that it has not been proven that hyponatremia correction can reduce mortality, these data further confirmed the clear relationship between hyponatremia and increased mortality in cancer patients.

An Italian study performed in Ancona analyzed 105 consecutive cancer patients, affected by gastrointestinal, lung, breast, female genital tract, renal, brain tumors, and sarcoma, hospitalized during a 6 month period ${ }^{[2]}$. 
Median overall survival (OS) from the day of hospitalization was 50 days. Hyponatremic patients had a significantly reduced OS $(P=0.0255)$ compared to normonatremic patients. In addition, the authors reported that the presence of metastases was associated with a reduced OS $(P=0.0418)$. Similar results were obtained by Castillo et al. ${ }^{[23]}$, who analyzed a population of patients with lymphoma, breast cancer, colorectal cancer, small cell and non-small cell lung cancer. Hyponatremia was negatively associated with OS in all types of tumor, but the highest HRs were found in lymphoma (HR 4.5, $P<0.01$ ) and in breast cancer (HR 3.7, $P<0.1)$.

In a retrospective cohort study, performed in a single center, 204 cancer patients affected by hyponatremia secondary to the syndrome of inappropriate anti-diuresis (SIAD), which is the most common cause of hyponatremia in oncology, were selected. Malignancies included lymphoma, leukemia, colorectal, breast, lung, pancreas, prostate, head and neck, bladder, esophagus, gastric cancer. About $75 \%$ of patients had malignancy-associated SIAD, whereas in roughly $25 \%$ SIAD was due to other etiologies (e.g., medications, pulmonary infections, pain or nausea). The authors found that patients with malignancy-associated SIAD had a significantly shorter median survival (58 days vs. 910 days, $P<0.001$ ). The authors hypothesized that the cause of SIAD in cancer patients might represent a useful prognostic factor ${ }^{[24]}$.

A correlation between hyponatremia and a negative outcome was found also in patients with terminal cancer. A retrospective observational study conducted in a tertiary hospital palliative care unit in the Republic of Korea reported that, in addition to serum C-reactive protein (HR 1.22; $P<0.001)$ and Palliative Performance Scale (HR 0.69, $P<0.001)$, serum $\left[\mathrm{Na}^{+}\right] \leq 125 \mathrm{mmol} / \mathrm{L}$ was associated with a reduced survival time $(\mathrm{HR}=1.91 ; P<0.001)$ among 576 terminally ill patients (pancreatic/hepatobiliary, gastric, colorectal, lung cancer $)^{[25]}$.

In a retrospective study performed at a University Hospital in China, among patients affected by nasopharyngeal carcinoma, glioma or oral cancer, that had been subjected to radiotherapy and developed radiation-induced brain necrosis, the risk of progression (i.e., increase of edema area $\geq 25 \%$ on the MRI) was three-fold higher in patients with hyponatremia compared to those with normonatremia. Thus, the authors claimed that hyponatremia may be considered as a potential predictor for the progression of radiationinduced brain necrosis and recommended that hyponatremia is appropriately managed also in these patients $^{[26]}$.

Other authors addressed their attention to a different subset of cancer patients, i.e., those who develop spontaneous tumor lysis syndrome, that induces acute uric acid nephropathy. This is a rare, yet potentially fatal complication in cancer patients, and it is important to determine the relevance of potential prognostic predictors. Although only 12 patients developed this condition among the 1,073 patients admitted with acute renal failure to a single hospital during a period of four years, hyponatremia and hypoalbuminemia on the first day of admission were the best predictors of poor prognosis ${ }^{[27]}$.

An interesting retrospective cohort study performed in a primary care setting in Copenhagen, Denmark, examined another issue, i.e., the predictive role of hyponatremia on the subsequent development of cancer. This type of analysis is facilitated in countries, like Denmark, which have a National Patient Registry. The authors showed that there is a level-dependent increased risk to develop cancer in patients with hyponatremia. The cumulative incidence increased in patients with mild (serum $\left[\mathrm{Na}^{+}\right]$130-135 mmol/L), moderate (125-129 mmol/ L) or severe hyponatremia (<125 mmol/L) [HR 1.32 (95\%CI: 1.26-1.39), 1.31 (95\%CI: 1.17-1.47), 1.77 (95\%CI: 1.392.24), respectively] at 12 months, compared to normonatremic subjects. When different cancers types were analyzed separately, this finding was confirmed in head and neck, pulmonary and gastrointestinal cancer ${ }^{[28]}$.

Some studies on the role of hyponatremia in cancer have addressed specific cancer types. With regard to renal cell carcinoma (RCC), 212 newly diagnosed patients with localized RCC undergoing nephrectomy were 
recruited in a study aiming to examine the prognostic value of hematologic and biochemical parameters, and other tumor-related factors. Multivariate analyses showed that preoperative serum $\left[\mathrm{Na}^{+}\right]$was an independent predictor of OS and disease-free survival, when considered either as a continuous variable or when patients were grouped based on a cut-off of serum $\left[\mathrm{Na}^{+}\right]$of $139 \mathrm{mmol} / \mathrm{L}$ (median value). The estimates of 5-year OS were 67.6\% (95\%CI: 54.2-80.9) and 44.3\% (95\%CI: 32.8-55.8) for patients with serum $\left[\mathrm{Na}^{+}\right]$above or below $139 \mathrm{mmol} / \mathrm{L}$ respectively ${ }^{[29]}$. A Danish study addressed patients with metastatic RCC, divided into two independent cohorts of 120 patients/each. In each cohort $20 \%$ and $14 \%$ of patients had hyponatremia. Patients with hyponatremia at baseline had a median OS of 5.5 and 4.8 months in the two cohorts, whereas patients with normonatremia at baseline had a median OS of 18.6 and 16.9 months, respectively ${ }^{[30]}$. In multivariate analysis, hyponatremia proved to be an independent prognostic factor (HR 1.90, 95\% CI: 1.1-3.2, $P=0.014$ ), together with increased neutrophils (HR 1.75, CI: 1.1-2.8, $P=0.018$ ), lactate dehydrogenase $>1.5$ ULN (HR 2.09, 95\%CI: 1.3-3.3, $P=0.002)$, and number of metastatic sites $(+3)($ HR 1.92, 95\%CI: 1.3-2.9, $P=$ 0.003). Finally, hyponatremia was significantly associated with lack of response to treatment in both cohorts. A more recent Japanese study confirmed that hyponatremia appears to be a powerful prognostic predictor in patients with metastatic RCC treated with tyrosine-kinase inhibitors following radical nephrectomy ${ }^{[31]}$. In this study both progression-free survival (PFS) and OS were significantly lower in hyponatremic patients (median 10.0 and 20.9 months, respectively) than in normonatremic patients (median 28.4 and 38.5 months, respectively).

Reduced serum $\left[\mathrm{Na}^{+}\right]$was indicated as a negative prognostic marker also in patients with gastric cancer and bone marrow metastases [HR 4.57; 95\%CI: 1.99-10.52; $P<0.001]^{[32]}$ and in patients with hepatocellular carcinoma ${ }^{[33]}$.

\section{WHY IS HYPONATREMIA ASSOCIATED WITH A WORSE OUTCOME IN CANCER PATIENTS?}

Clinical data clearly indicate that hyponatremia may be viewed as a predictor of a negative outcome in cancer patients. Admittedly, one open question is whether the reduced PFS and OS, described by a number of publications, is directly attributable to the electrolyte disorder itself or might be a sign of the severity of the underlying disease. With regard to this point, a Belgian prospective study on hyponatremia in cancer patients, published almost 20 years ago, suggested that the increase mortality rate observed in cancer patients with hyponatremia ( $19.5 \%$ vs $6.3 \%$ in normonatremic partients) was not apparently due to reduced serum $\left[\mathrm{Na}^{+}\right]$, thus indicating that hyponatremia was to be considered as a marker of general debility in advanced disease ${ }^{[34]}$. A retrospective review (entitled "Mortality and serum sodium: do patients die from or with hyponatremia?") of the medical records of patients admitted to a teaching hospital in Rochester, NY, for different pathologies and who died after developing hyponatremia, also suggested that the main determinant of the observed deaths was likely the severity of the underlying illness rather than the degree of hyponatremia ${ }^{[35]}$. Similarly, a retrospective study that included patients diagnosed with SIAD in a community hospital in Israel showed that long-term survival was determined by SIAD etiology rather than by hyponatremia severity ${ }^{[36]}$.

Recently, a study performed at two teaching hospitals in Italy and one teaching hospital in England demonstrated that serum $\left[\mathrm{Na}^{+}\right]$normalization during first-line therapy is an independent prognostic factor for OS and PFS in patients with non-small cell lung cancer ${ }^{[37]}$. Similarly, normalization of serum $\left[\mathrm{Na}^{+}\right]$was associated with a better prognosis in patients with metastatic RCC treated with everolimus ${ }^{[38]}$. These findings are in agreement with the results of a meta-analysis that analyzed articles reporting the outcome of patients with different diseases in which hyponatremia had been corrected. The meta-analysis indicated that any improvement of hyponatremia was associated with a reduced risk of overall mortality $[\mathrm{HR}=0.57(0.40-0.81)]^{[39]}$.

Anyway, this issue is still a matter of debate and prospective randomized studies in cancer patients with hyponatremia, aiming to evaluate whether correction of hyponatremia can counteract the progression of the disease, are necessary. 
Interestingly, some basic research studies have evaluated whether a microenvironment with a low $\left[\mathrm{Na}^{+}\right]$may affect cell proliferation and invasion ability. A study performed in prostate cancer cells in vitro demonstrated for instance that the exposure to a slightly hypertonic milieu induced a dormant state. Cell dormancy represents a limiting step of the metastatic process by preventing the proliferation of isolated cells outside the primary tumor. Conversely, the authors demonstrated that in the presence of a hypotonic milieu, obtained for instance by reducing $\left[\mathrm{Na}^{+}\right]$in the culture medium, cell cloning significantly increased ${ }^{[40]}$. Another study showed that low $\left[\mathrm{Na}^{+}\right]$reduced neuroblastoma cell adhesion and increased invasion ability ${ }^{[41]}$. A micro-array analysis was performed, in order to analyze the gene expression pattern of cells exposed to low $\left[\mathrm{Na}^{+}\right]$compared to normal $\left[\mathrm{Na}^{+}\right]$. Among the genes that had different expression levels in the presence of reduced $\left[\mathrm{Na}^{+}\right]$, the heme-oxigenase 1 gene (HMOX-1), a marker of oxidative stress, was the gene with the greatest variation. In fact, $\mathrm{HMOX}-1$ gene expression showed a 200 -fold increase in cells exposed to low $\left[\mathrm{Na}^{+}\right]$. Immunocytochemistry for HMOX-1 protein confirmed these results. It is known that oxidative stress favors carcinogenesis, cancer growth and invasion, angiogenesis, and overall creates a permissive environment for cancer cells ${ }^{[42]}$. Accordingly, selective inhibition of HMOX-1 has been proposed as a therapeutic target for cancer treatment ${ }^{[43]}$. Although additional studies are needed in order to confirm these data, in view of the above described results from basic research it can be hypothesized that also in vivo low $\left[\mathrm{Na}^{+}\right]$might promote cancer cell progression through the same mechanisms. If so, then we might probably say that patients die not only with hyponatremia, but also for hyponatremia.

\section{CONCLUSION}

In recent years evidence indicating that hyponatremia is a predictor of a worse outcome in cancer patients has accumulated. There is emerging evidence, mainly from basic research studies at this time, that hyponatremia may by itself increase the risk of mortality of patients, rather than being a simple bystander of the progression of the disease. However, robust confirmatory data from clinical practice are certainly needed in order to definitively validate the hypothesis that cancer patients may die for hyponatremia.

Meanwhile, the author is truly convinced that it is worth to correct hyponatremia in patients, including cancer patients, because of the overall beneficial effect on the quality of life, which includes clinical improvement, such as amelioration of neurocognitive and motor performance, reduced length of stay in the hospital and re-admission probability ${ }^{[44-46]}$.

\section{DECLARATIONS}

\section{Authors' contributions}

The author contributed solely to the article.

\section{Availability of data and materials}

Not applicable.

\section{Financial support and sponsorship}

None.

\section{Conflicts of interest}

The author declares that there are no conflicts of interest.

\section{Ethical approval and consent to participate}

Not applicable.

\section{Consent for publication}

Not applicable. 


\section{Copyright}

(c) The Author(s) 2019.

\section{REFERENCES}

1. Upadhyay A, Jaber BL, Madias NE. Epidemiology of hyponatremia. Semin Nephrol 2009;29:227-38.

2. Kremeike K, Wetter RML, Burst V, Voltz1 R, Kuhr K, et al. Prevalence of hyponatremia in inpatients with incurable and life-limiting diseases and its association with physical symptoms-a retrospective descriptive study. Support Care Cancer 2018;26:213-22.

3. Wald R, Jaber BL, Price LL. Impact of hospital-associated hyponatremia on selected outcomes. Arch Intern Med 2010;170:294-302.

4. Klein L, O'Connor CM, Leimberger JD, Gattis-Stough W, Pinha IL, et al. OPTIME-CHF investigators lower serum sodium is associated with increased short-term mortality in hospitalized patients with worsening heart failure: results from the outcomes of a prospective trial of intravenous milrinone for exacerbations of chronic heart failure (OPTIME-CHF) study. Circulation 2005;111:2454-60.

5. Rusinaru D, Tribouilloy C, Berry C, Richards AM, Whalley GA, et al. MAGGIC investigators: relationship of serum sodium concentration to mortality in a wide spectrum of heart failure patients with preserved and with reduced ejection fraction: an individual patient data meta-analysis $(\dagger)$ : meta-analysis global group in chronic heart failure (MAGGIC). Eur J Heart Fail 2012;14:1139-46.

6. Price JF, Kantor PF, Shaddy RE, Rossano JW, Goldberg JF, et al. Incidence, severity, and association with adverse outcome of hyponatremia in children hospitalized with heart failure. Am J Cardiol 2016;118:1006-10.

7. Waikar SS, Mount DB, Curhan GC. Mortality after hospitalization with mild, moderate, and severe hyponatremia. Am J Med 2009;122:857-65.

8. Choi JS, Kim CS, Bae EH, Ma SK, Ahn YK, et al. Prognostic impact of hyponatremia occurring at various time points during hospitalization on mortality in patients with acute myocardial infarction. Medicine (Baltimore) 2017;96:e7023.

9. Zilberberg MD, Exuzides A, Spalding J, Foreman A, Jones AG, et al. Hyponatremia and hospital outcomes among patients with pneumonia: a retrospective cohort study. BMC Pulm Med 2008;8:16.

10. Kim WR, Biggins SW, Kremers WK, Wiesner RH, Kamath PS, et al. Hyponatremia and mortality among patients on the liver-transplant waiting list. N Engl J Med 2008;359:1018-26.

11. Fortune B, Cardenas A. Ascites, refractory ascites and hyponatremia in cirrhosis. Gastroenterol Rep (Oxf) 2017;5:104-12.

12. Sun L, Hou Y, Xiao Q, Du Y. Association of serum sodium and risk of all-cause mortality in patients with chronic kidney disease: a metaanalysis and sysematic review. Sci Rep 2017;7:15949.

13. Zhou XY, Chen HL, Ni SS. Hyponatremia and short-term prognosis of patients with acute pulmonary embolism: a meta-analysis. Int J Cardiol 2017;227:251-6.

14. Carcel C, Sato S, Zheng D, Heeley E, Arima H, et al. Prognostic significance of hyponatremia in acute intracerebral hemorrhage: pooled analysis of the intensive blood pressure reduction in acute cerebral hemorrhage trial studies. Crit Care Med 2016;44:1388-94.

15. Terzian C, Frye EB, Piotrowski ZH. Admission hyponatremia in the elderly: factors influencing prognosis. J Gen Intern Med 1994;9:8991.

16. Stelfox HT, Ahmed SB, Khandwala F, Zygun D, Shahpori R, et al. The epidemiology of intensive care unit-acquired hyponatremia and hypernatraemia in medical-surgical intensive care units. Crit Care 2008;12:R162.

17. Martin JY, Goff BA, Urban RR. Preoperative hyponatremia in women with ovarian cancer: an additional cause for concern? Gynecol Oncol 2016;142:471-6.

18. Kagase A, Yamamoto M, Shimura T, Kodama A, Kano S, et al. Impact of pre-procedural hyponatremia on clinical outcomes after transcatheter aortic valve replacement: a propensity-matched analysis. Catheter Cardiovasc Interv 2018;92:E125-34.

19. Corona G, Giuliani C, Parenti G, Norello D, Verbalis JG, et al. Moderate hyponatremia is associated with increased risk of mortality: evidence from a meta-analysis. PLoS One 2013;8:e80451.

20. Elsayem A, Mori M, Parsons HA, Munsell MF, Hui D, et al. Predictors of inpatient mortality in an acute palliative care unit at a comprehensive cancer center. Support Care Cancer 2010;18:67-76.

21. Doshi SM, Shah P, Lei X, Lahoti A, Salahudeen AK. Hyponatremia in hospitalized cancer patients and its impact on clinical outcomes. Am J Kidney Dis 2012;59:222-8.

22. Berardi R, Caramanti M, Castagnani M, Guglielmi S, Marcucci F, et al. Hyponatremia is a predictor of hospital length and cost of stay and outcome in cancer patient. Support Care Cancer 2015;23:3095-101.

23. Castillo JJ, Glezerman IG, Boklage SH, Chiodo J 3rd, Tidwell BA, et al. The occurrence of hyponatremia and its importance as a prognostic factor in a cross-section of cancer patients. BMC Cancer 2016;16:564.

24. Goldvaser H, Rozen-Zvi B, Yerushalmi R, Gafter-Gvili A, Lahav M, et al. Malignancy associated SIADH: characterization and clinical implications. Acta Oncol 2016;55:1190-5.

25. Yoon J, Ahn SH, Lee YJ, Kim CM. Hyponatremia as an independent prognostic factor in patients with terminal cancer. Support Care Cancer 2015;23:1735-40.

26. Liao H, Zhu Z, Rong X, Wang H, Peng Y. Hyponatremia is a potential predictor of progression in radiation-induced brain necrosis: a retrospective study. BMC Neurol 2018;18:130.

27. Hsu HH, Chen YC, Tian YC, Chan YL, Kuo MC, et al. Role of serum sodium in assessing hospital mortality in cancer patients with spontaneous tumour lysis syndrome inducing acute uric acid nephropathy. Int J Clin Pract 2009;63:751-6.

28. Selmer C, Madsen JC, Torp-Pedersen C, Gislason GH, Faber J. Hyponatremia, all-cause mortality, and risk of cancer diagnoses in the primary care setting: a large population study. Eur J Intern Med 2016;36:36-43.

29. Vasudev NS, Brown JE, Brown SR, Rafiq R, Morgan R, et al. Prognostic factors in renal cell carcinoma: association of preoperative sodium concentration with survival. Clin Cancer Res 2008;14:1775-81.

30. Jeppesen AN, Jensen HK, Donskov F, Marcussen N, von der Maase H. Hyponatremia as a prognostic and predictive factor in metastatic renal cell carcinoma. Br J Cancer 2010;102:867-72. 
31. Furukawa J, Miyake H, Kusuda Y, Fujisawa M. Hyponatremia as a powerful prognostic predictor for Japanese patients with clear cell renal cell carcinoma treated with a tyrosine kinase inhibitor. Int J Clin Oncol 2015;20:351-7.

32. Kim HS, Yi SY, Jun HJ, Lee J, Park JO, et al. Clinical outcome of gastric cancer patients with bone marrow metastases. Oncology 2007;73:192-7.

33. Hsu CY, Lee YH, Hsia CY, Huang YH, Su CW, et al. Performance status in patients with hepatocellular carcinoma: determinants, prognostic impact, and ability to improve the barcelona clinic liver cancer system. Hepatology 2013;57:112-9.

34. Berghmans T, Paesmans M, Body JJ. A prospective study on hyponatraemia in medical cancer patients: epidemiology, aetiology and differential diagnosis. Support Care Cancer 2000;8:192-7.

35. Chawla A, Sterns RH, Nigwekar SU, Cappuccio JD. Mortality and serum sodium: do patients die from or with hyponatremia. Clin J Am Soc Nephrol 2011;6:960-5.

36. Shepshelovich D, Leibovitch C, Klein A, Zoldan S, Milo G, Shochat T et al. The syndrome of inappropriate antidiuretic hormone secretion: distribution and characterization according to etiologies. Eur J Intern Med 2015;26:819-24.

37. Berardi R, Santoni M, Newsom-Davis T, Caramanti M, Rinaldi S, et al. Hyponatremia normalization as an independent prognostic factor in patients with advanced non-small cell lung cancer treated with first-line therapy. Oncotarget. 2017;8:23871-9.

38. Penttilä P, Bono P, Peltola K, Donskov F. Hyponatremia associates with poor outcome in metastatic renal cell carcinoma patients treated with everolimus: prognostic impact. Acta Oncol 2018;57:1580-5.

39. Corona G, Giuliani C, Verbalis JG, Forti G, Maggi M, et al. Hyponatremia improvement is associated with a reduced risk of mortality: evidence from a meta-analysis. PLoS One 2015;10:e124105.

40. Havard M, Dautry F, Tchénio T. A dormant state modulated by osmotic pressure controls clonogenicity of prostate cancer cells. J Biol Chem 2011;286:44177-86.

41. Benvenuti S, Deledda C, Luciani P, Modi G, Bossio A, et al. Low extracellular sodium causes neuronal distress independently of reduced osmolality in an experimental model of chronic hyponatremia. Neuromolecular Med. 2013;15:493-503.

42. Chau LY. Heme oxygenase-1: emerging target of cancer therapy. J Biomed Sci 2015;22:22.

43. Yadav B, Greish K. Selective inhibition of hemeoxygenase-1 as a novel therapeutic target for anticancer treatment. J Nanomedic Nanotechnol 2011;S4:5.

44. Bhandari S, Peri A, Cranston I, McCool R, Shaw A, Glanville J, et al. A systematic review of known interventions for the treatment of chronic nonhypovolaemic hypotonic hyponatraemia and a meta-analysis of the vaptans. Clin Endocrinol (Oxf) 2017;86:761-71.

45. Schrier RW, Gross P, Gheorghiade M, Berl T, Verbalis JG, et al. Tolvaptan, a selective oral vasopressin V2-receptor antagonist, for hyponatremia. N Engl J Med 2006;355:2099-112.

46. Verbalis JG, Adler S, Schrier RW, Berl T, Zhao Q, et al. Efficacy and safety of oral tolvaptan therapy in patients with the syndrome of inappropriate antidiuretic hormone secretion. Eur J Endocrinol 2011;164:725-32. 